\title{
Respuesta hemodinámica precoz a los corticoides en el shock séptico
}

\author{
JUAN A. LLOMPART-POU ${ }^{a, b}$, JOAN M. RAURICHa, JORDI IBÁÑEZa , MARÍA RIESCOc \\ Y JOSÉ I. AYESTARÁNa

\begin{abstract}
aServicio de Medicina Intensiva. Hospital Universitario Son Dureta. Palma de Mallorca. Islas Baleares. España. Islas Baleares. España.
\end{abstract}

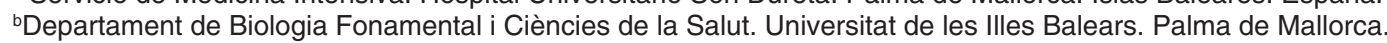

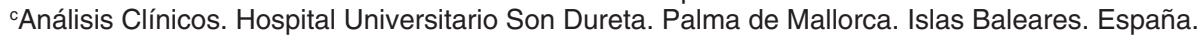

Objetivo. El tratamiento con corticoides a dosis bajas (LDST) en los pacientes con shock séptico tratados con soporte vasoactivo se ha asociado a una disminución del tiempo de shock. Los pacientes con cortisol basal disminuido o con respuesta anormal a la estimulación suprarrenal deberían tener un mayor beneficio con los LDST, por lo que hemos estudiado la respuesta hemodinámica precoz en pacientes con shock séptico tratados o no con LDST.

Diseño. Estudio retrospectivo.

Ámbito. Unidad de cuidados intensivos (UCI) en un hospital universitario de tercer nivel.

Pacientes. Estudiamos a 96 pacientes ingresados en la $\mathrm{UCI}$, distribuidos en dos grupos de 48 pacientes según hubieran recibido corticoides (grupo A) o no (grupo B), apareados según los valores de cortisol basal y los requerimientos de noradrenalina.

Intervenciones. A todos los pacientes, se realizó un test de estimulación suprarrenal con $250 \mu \mathrm{g}$ de corticotrofina en las primeras $72 \mathrm{~h}$ del inicio del shock.

Variables principales de interés. Cortisol basal, incremento de cortisol, cortisol máximo, reducción de la dosis de noradrenalina, duración del shock.

Resultados. Ambos grupos fueron comparables. Las cifras de cortisol basal antes del test de estimulación suprarrenal y el cortisol máximo fueron similares en ambos grupos. No hubo diferencias significativas entre ambos grupos en la dosis

Correspondencia: Dr. J.A. Llompart-Pou.

Servicio de Medicina Intensiva. Hospital Universitario Son Dureta. Andrea Doria, 55. 07014 Palma de Mallorca. Illes Balears. España. Correo electrónico: jallompart@hsd.es

Manuscrito aceptado el 3-3-2008. de noradrenalina antes y a las $24 \mathrm{~h}$ tras la estimulación suprarrenal. La reducción en la dosis de noradrenalina a las $24 \mathrm{~h}$ no se correlacionó con las cifras de cortisol basal ni con la respuesta a la estimulación suprarrenal.

Conclusiones. El tratamiento con LDST no se asoció a una mejoría hemodinámica a las 24 h, independientemente de las cifras de cortisol basal y de la respuesta a la estimulación suprarrenal.

PALABRAS CLAVE: Cortisol. Shock séptico. Respuesta hemodinámica. Insuficiencia suprarrenal. Corticoides.

\section{IMMEDIATE HEMODYNAMIC RESPONSE TO STEROID TREATMENT IN SEPTIC SHOCK}

Objective. Treatment with low dose steroids (LDST) in patients with septic shock treated with vasoactive agents has been related to earlier shock reversal. Patients with low baseline cortisol and a blunted response to the corticotropin test are more likely to benefit from LDST, so we compared the immediate hemodynamic response in patients with septic shock who received LDST with that of those who did not receive LDST.

Design. Retrospective study.

Scope. Intensive Care Unit (ICU) of a tertiary university hospital.

Patients. We studied 96 patients admitted to the ICU. Patients were classified in two groups of 48 patients: group A received LDST and group B did not; patients were matched according to baseline cortisol levels and norepinephrine requirements.

Interventions. All patients underwent a short corticotropin test (250 $\mu \mathrm{g} \mathrm{ACTH)} \mathrm{within} 72 \mathrm{~h}$ of septic shock onset. 
Main variables of interest. Baseline cortisol, delta cortisol, peak cortisol, norepinephrine reduction after LDST, and duration of shock.

Results. The two groups were comparable: baseline and stimulated cortisol levels before corticotropin test were similar, and there were no differences in the norepinephrine dose before and $24 \mathrm{~h}$ after testing adrenal response $(p=0.98$ and $p$ $=0.53$, respectively). Norepinephrine reduction at $24 \mathrm{~h}$ after testing was not correlated with baseline cortisol or with adrenal response to the corticotropin test.

Conclusions. LDST was not associated to improved 24-hour hemodynamic response, irrespective of the baseline and stimulated cortisol levels.

KEY WORDS: Cortisol. Septic shock. Hemodynamic response. Adrenal insufficiency. Steroids.

\section{INTRODUCCIÓN}

Las guías de la Surviving Sepsis Campaign recomiendan el uso de hidrocortisona a dosis de 200-300 $\mathrm{mg}$ /día (low dose steroid therapy [LDST]) en los pacientes con shock séptico que necesitan tratamiento con fármacos vasoactivos ${ }^{1}$.

Diversos estudios indican que el cortisol, a través de diversos mecanismos, es determinante para mantener la contractilidad cardíaca, el tono vascular y la presión arterial ${ }^{2-4}$. Estudios recientes indican que los enfermos con shock séptico dependientes de soporte vasoactivo deben ser tratados con LDST para conseguir una mejoría hemodinámica y una reducción del tiempo de $\operatorname{shock}^{1,5-8}$. Sin embargo, no está bien determinado qué cifras de cortisol deben considerarse óptimas en el shock séptico (se han propuesto valores basales entre 10 y $34 \mu \mathrm{g} / \mathrm{dl}$ ) y, además, no se ha precisado con exactitud si el tratamiento con LDST debe realizarse según los valores basales de cortisol o dependiendo del resultado tras una prueba de estimulación dinámica suprarrenal ${ }^{9-11}$.

Diversos autores recomiendan iniciar el tratamiento con LDST en los pacientes con inestabilidad hemodinámica, independientemente de las cifras de cortisol $^{12,13}$. Una adecuada respuesta hemodinámica precoz sería el mejor indicador de disfunción suprarrenal $^{12,13}$. Recientemente hemos revisado el impacto de los LDST en los pacientes con shock séptico ingresados en nuestra unidad de cuidados intensivos (UCI) en una muestra de 203 pacientes, y no hemos hallado relación con la mortalidad ni con el tiempo de shock $^{14}$. Por todo ello, consideramos que no se ha establecido con claridad qué grupos de pacientes podrían beneficiarse del tratamiento con LDST.

Los pacientes con cifras bajas de cortisol basal y los que no responden a la estimulación suprarrenal con corticotrofina cuando se los trata con LDST deberían presentar una mejor respuesta hemodinámica que los no tratados con LDST. Por lo tanto, hemos estudiado la respuesta hemodinámica a las 24 h en pacientes con shock séptico, tratados o no con LDST.

\section{PACIENTES Y MÉTODOS}

Se seleccionó a 162 pacientes de una base de datos de pacientes con shock séptico recogida de modo retrospectivo. Entre ellos, 103 fueron tratados con LDST y 59 no recibieron corticoides. La decisión de tratar o no con LDST correspondió al médico intensivista responsable de cada paciente. Se excluyó del análisis a los pacientes que previamente habían recibido medicación que pudiera interferir en el metabolismo del cortisol (corticoides previos, etomidato, ketoconazol) y a las pacientes embarazadas. Se apareó a los restantes para que recibieran LDST o no, de acuerdo con intervalos de cortisol basal de $5 \mu \mathrm{g} / \mathrm{dl}$ y a las dosis de noradrenalina (NA) en diferencias inferiores al $20 \%$, con lo que resultó un total de 96 pacientes. De este modo, se analizó retrospectivamente a 96 pacientes con shock séptico ingresados en la UCI de nuestro hospital entre abril de 2003 y noviembre de 2005. El comité de investigación de nuestro centro aprobó el estudio y consideró innecesario obtener el consentimiento informado, al tratarse de un estudio observacional y retrospectivo.

Todos los pacientes recibieron NA para mantenerse estables hemodinámicamente. La función suprarrenal se evaluó utilizando el test de estimulación suprarrenal con $250 \mu \mathrm{g}$ de corticotrofina. Se trató con LDST (50 mg de hidrocortisona intravenosa cada $6 \mathrm{~h}$ y $0,05 \mathrm{mg}$ de fludrocortisona cada $24 \mathrm{~h}$ por vía oral cuando la vía digestiva funcionaba correctamente) a 48 pacientes (grupo A), al igual que en el estudio de Annane et $\mathrm{al}^{7}$. Como grupo control (grupo B), seleccionamos a 48 pacientes con shock séptico a los que se realizó un test de estimulación suprarrenal pero no recibieron tratamiento con LDST.

El tratamiento hemodinámico de estos pacientes siguió un protocolo preestablecido, previamente detallado ${ }^{14}$, que incluye la optimización de la volemia, el empleo de NA para conseguir una presión arterial media $(\mathrm{PAM})>65 \mathrm{mmHg}$ y soporte inotrópico con dobutamina si se detectaba disfunción cardíaca asociada. Uno de los objetivos era conseguir una diuresis $>0,5 \mathrm{ml} / \mathrm{kg} / \mathrm{h}$.

Se documentó la dosis de NA necesaria antes y a las 24 h de la realización del test de estimulación suprarrenal, así como el tiempo total de tratamiento con NA. La gravedad de los pacientes se evaluó con los siguientes índices de gravedad: Simplified Acute Physiology Score II (SAPS-II) ${ }^{15}$, Acute Physiology and Chronic Health Evaluation II (APACHE II) ${ }^{16}$ y el Sepsis-related Organ Failure Assesment (SOFA) ${ }^{17}$ a las $24 \mathrm{~h}$ de ingreso y el valor máximo durante la primera semana del shock séptico. También se recogieron datos respecto al número de pacientes tratados con ventilación mecánica, terapias de reemplazo renal y empleo de drotrecogina alfa. Además, se documentó el número de pacientes que recibieron tratamiento antibiótico inadecuado y el balance hídrico de 
las primeras $24 \mathrm{~h}$, sin contabilizar las pérdidas insensibles.

De todos los pacientes se obtuvieron muestras de sangre periférica a través de un catéter arterial para determinar el cortisol basal y a los 30 y los 60 min tras la estimulación de la glándula suprarrenal con $250 \mu \mathrm{g}$ de corticotrofina (Synacthen) durante las primeras $72 \mathrm{~h}$ de la instauración del shock séptico.

Las muestras se recogieron en tubos sin anticoagulante para determinar cortisol, se centrifugaron inmediatamente y se almacenaron a $-20^{\circ} \mathrm{C}$ hasta su análisis. El cortisol sérico se analizó mediante un análisis de inmunoquimioluminiscencia directa en analizador Advia, Centaour (Siemens, M.S. Diagnostics). Los valores de referencia en sujetos sanos en nuestro laboratorio son 4,3-24,4 $\mu \mathrm{g} / \mathrm{dl}$. El límite de detección es de $0,2 \mu \mathrm{g} / \mathrm{dl}$, la recuperación a distintos niveles se sitúa entre el 86,2 y el $114 \%$, con una media del $101 \%$. La imprecisión intraanalítica oscila entre el 2,89 y el $3,62 \%$, y entre análisis, entre el 1,86 y el $5,45 \%$ en relación con el nivel analizado.

Se diagnosticó shock séptico de acuerdo con los criterios de la International Consensus Conference Definition $^{18}$. La insuficiencia suprarrenal relativa (ISR) se diagnosticó en los pacientes con un incremento $<9 \mu \mathrm{g} / \mathrm{dl}$ de sus concentraciones de cortisol respecto a las basales tras la estimulación con corticotrofina ${ }^{7}$. Se consideró cese del shock cuando se suspendieron los fármacos vasoactivos ${ }^{5,6}$. La respuesta hemodinámica precoz se evaluó a las 24 h de la estimulación glandular suprarrenal.

El desenlace principal del estudio es la variación en la dosis de noradrenalina a las $24 \mathrm{~h}$ de iniciarse o no el tratamiento con corticoides. Como variables secundarias, se evaluó la mortalidad hospitalaria y el tiempo de shock.

\section{Análisis estadístico}

Los datos se presentan como media y desviación estandar. Las diferencias entre los grupos se analizaron utilizando la prueba de la $\chi^{2}$ o la exacta de Fisher para las variables categóricas y la de la t de Student o la U de Mann-Whitney para las variables continuas. Evaluamos la duración del shock séptico utilizando el método de Kaplan-Meier y comparamos los grupos con el log-rank test. Un valor de $\mathrm{p}<0,05$ fue indicativo de significación estadística. Los datos se analizaron utilizando SPSS statistical package version 13.0 (SPSS Inc., Chicago).

\section{RESULTADOS}

Los pacientes de ambos grupos presentaban en el momento de ser incluidos iguales características clínicas y demográficas, gravedad del shock séptico, comorbilidades, incidencia de pacientes posquirúrgicos $\mathrm{y}$ foco infeccioso (tabla 1 ).

El tiempo transcurrido entre el diagnóstico de shock séptico y la realización del test de estimulación suprarrenal fue de 1,5 \pm 2,6 días en el grupo tratado
TABLA 1. Características de los pacientes con shock séptico incluidos en el estudio

\begin{tabular}{|l|c|c|c|}
\hline & Grupo A $(\mathrm{n}=48)$ & Grupo B $(\mathrm{n}=48)$ & $\mathrm{p}$ \\
\hline Varones/mujeres & $33 / 15$ & $30 / 18$ & 0,52 \\
Edad (años) & $62 \pm 15$ & $61 \pm 14$ & 0,66 \\
Peso (kg) & $75 \pm 15$ & $76 \pm 19$ & 0,72 \\
SAPS II & $46 \pm 13$ & $43 \pm 15$ & 0,24 \\
APACHE II & $21 \pm 5$ & $20 \pm 8$ & 0,76 \\
SOFA (primeras 24 h) & $11,1 \pm 2,6$ & $10,7 \pm 3,3$ & 0,52 \\
Comorbilidad & & & \\
Diabetes mellitus & $7(14,6)$ & $11(22,9)$ & 0,3 \\
EPOC & $12(25)$ & $10(20,8)$ & 0,63 \\
Afección cardíaca & $10(20,8)$ & $8(16,7)$ & 0,6 \\
Hepatopatía crónica & $4(8,3)$ & $6(12,5)$ & 0,5 \\
Insuficiencia renal crónica & $3(6,2)$ & $4(8,3)$ & 1 \\
Neoplasias & $8(16,7)$ & $6(12,5)$ & 0,56 \\
Uso de corticoides <1 año & $6(12,5)$ & $5(10,4)$ & 0,75 \\
Foco infeccioso & & & 0,41 \\
Respiratorio & $27(56,2)$ & $25(52,1)$ & \\
Abdominal & $15(31,2)$ & $14(29,2)$ & \\
Urinario & $2(4,2)$ & $6(12,5)$ & \\
Otro & $4(8,3)$ & $3(6,2)$ & \\
Quirúrgicos & $15(31,3)$ & $12(25)$ & 0,5 \\
\hline
\end{tabular}

APACHE: Acute Physiology and Chronic Health Evaluation; EPOC: enfermedad pulmonar obstructiva crónica; SAPS: Simplified Acute Physiology Score; SOFA: Sepsis-related Organ Failure Assessment.

Grupo A: pacientes tratados con corticoides; grupo B: grupo control.

Los resultados expresan media \pm desviación estándar o n (\%)

con LDST y 1,3 \pm 2,1 días en el grupo control ( $\mathrm{p}=$ $0,61)$. Ambos grupos presentaron similares valores de cortisol basal, valor máximo de cortisol tras administrar $250 \mu \mathrm{g}$ de corticotrofina e incidencia de ISR (tabla 2). Las cifras de albúmina sérica el día en que se realizó la estimulación suprarrenal fueron similares en ambos grupos (grupo A, 20,9 $\pm 4,7 \mathrm{~g} / \mathrm{dl}$; grupo $\mathrm{B}, 21,5 \pm 5,2 \mathrm{~g} / \mathrm{dl} ; \mathrm{p}=0,52)$.

Las dosis de NA antes y a las 24 h de la estimulación suprarrenal fueron similares en ambos grupos, así como la diferencia de NA (tabla 3). La reducción de la dosis de NA a las 24 h no guardó relación con las cifras de cortisol basales (fig. 1) ni con la respuesta suprarrenal tras la estimulación con corticotrofina (tabla 4). Las curvas de Kaplan-Meier no demostraron diferencias entre ambos grupos en el tiempo de tratamiento con NA (mediana en el grupo A, 7 días; grupo $\mathrm{B}, 8$ días; $\mathrm{p}=0,99)$.

No hubo diferencias entre ambos grupos en la incidencia de pacientes tratados con ventilación mecá-

TABLA 2. Cortisol basal y tras estimulación glandular con $250 \mathrm{mg}$ de corticotrofina en ambos grupos

\begin{tabular}{|l|l|l|c|}
\hline & Grupo A $(\mathrm{n}=48)$ & Grupo B $(\mathrm{n}=48)$ & $\mathrm{p}$ \\
\hline Cortisol basal $(\mu \mathrm{g} / \mathrm{dl})$ & $22 \pm 12,3$ & $21,7 \pm 12$ & 0,92 \\
Cortisol $(\mu \mathrm{g} / \mathrm{dl})$ & $28,2 \pm 13,6$ & $28,9 \pm 12,1$ & 0,79 \\
$\quad \begin{array}{lll}\text { a los 30 min de HDCST } \\
\text { Cortisol }(\mu \mathrm{g} / \mathrm{dl})\end{array}$ & $29,7 \pm 13,7$ & $30,6 \pm 12,3$ & 0,74 \\
$\quad$ a los 60 min de HDCST & & & \\
$\begin{array}{l}\text { Diferencia de cortisol } \\
\quad \leq 9 \mu \mathrm{g} / \mathrm{dl} \text { tras HDCST }\end{array}$ & $31(64,6)$ & $24(50)$ & 0,18 \\
$\quad$ & & \\
\hline
\end{tabular}

HDCST: prueba de estimulación con altas dosis de corticotrofina.

Grupo A: pacientes tratados con corticoides; grupo B: grupo control.

Los datos expresan media \pm desviación estándar o n $(\%)$. 
TABLA 3. Dosis de noradrenalina antes y a las $24 \mathrm{~h}$ de la estimulación suprarrenal

\begin{tabular}{|l|c|c|c|}
\hline & Grupo A $(\mathrm{n}=48)$ & Grupo B $(\mathrm{n}=48)$ & $\mathrm{p}$ \\
\hline $\begin{array}{l}\text { Noradrenalina basal } \\
(\mu \mathrm{g} / \mathrm{kg} / \mathrm{min})\end{array}$ & $0,60 \pm 0,37$ & $0,59 \pm 0,35$ & 0,88 \\
$\begin{array}{c}\text { Noradrenalina a las 24 h } \\
(\mu \mathrm{g} / \mathrm{kg} / \mathrm{min})\end{array}$ & $0,41 \pm 0,38$ & $0,39 \pm 0,42$ & 0,53 \\
$\begin{array}{l}\text { Noradrenalina diferencia } \\
(\mu \mathrm{g} / \mathrm{kg} / \mathrm{min})\end{array}$ & $0,19 \pm 0,36$ & $0,20 \pm 0,36$ & 0,96 \\
\hline
\end{tabular}

HDCST: prueba de estimulación con altas dosis de corticotrofina.

Grupo A: pacientes tratados con corticoides; grupo B: grupo control.

Los datos expresan media \pm desviación estándar salvo que se indique otra cosa.

nica, terapias de reemplazo renal y drotrecogina alfa (tabla 5). Tampoco hallamos diferencias en el número de pacientes que recibieron tratamiento antibiótico inadecuado ni en el balance hídrico a las 24 h (tabla 5).

El SOFA máximo fue más elevado en el grupo A que en el grupo $\mathrm{B}(13,4 \pm 3,2$ y $12,0 \pm 3,5$ respectivamente; $p=0,054)$. No hallamos diferencias significativas en la mortalidad hospitalaria entre ambos grupos (el 54\% en el grupo A y el 52\% en el grupo B; p $=0,84)$.

\section{DISCUSIÓN}

Los resultados de nuestro estudio indican que los pacientes con shock séptico tratados con hidrocortisona, independientemente de las cifras basales de cortisol y la respuesta suprarrenal, no presentan una mejor respuesta hemodinámica a las 24 h que los que no reciben dicho tratamiento.

Los efectos de los corticoides en la respuesta cardiovascular, aunque conocidos, no están plenamente comprendidos $^{19}$. El cortisol contribuye a mantener la contractilidad cardíaca, el tono vascular y la presión

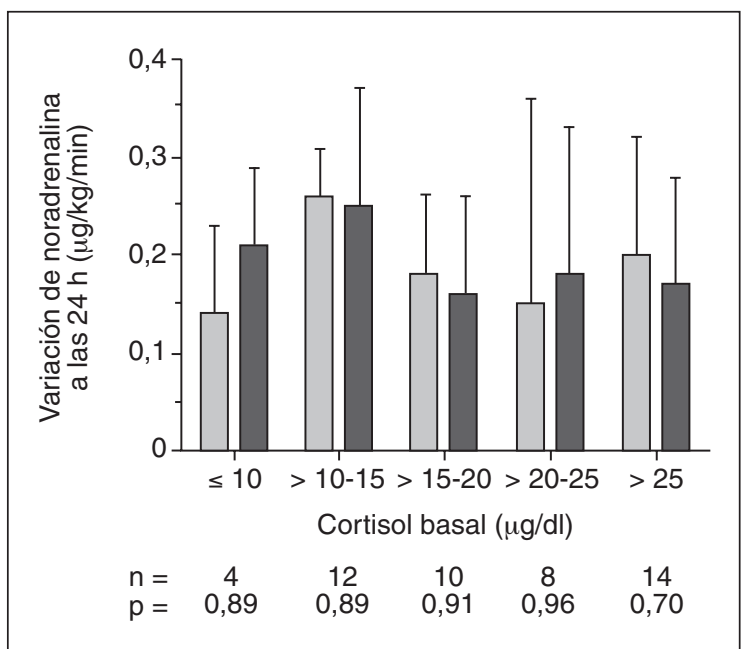

Figura 1. Variación en la dosis de noradrenalina las primeras 24 $h$ en los pacientes tratados con bajas dosis de corticoides (barras grises) y grupo control (barras negras), según las cifras basales de cortisol.
TABLA 4. Diferencias en la respuesta hemodinámica en los pacientes con y sin ISR, según recibieran o no tratamiento con corticoides

\begin{tabular}{|l|c|c|c|}
\hline & $\begin{array}{c}\text { Grupo A } \\
(\mathrm{n}=48)\end{array}$ & $\begin{array}{c}\text { Grupo B } \\
(\mathrm{n}=48)\end{array}$ & $\mathrm{p}$ \\
\hline $\begin{array}{c}\text { Diferencia de cortisol } \\
\leq 9 \mu \mathrm{g} / \mathrm{dl} \text { tras HDCST, } \mathrm{n}\end{array}$ & 31 & 25 & \\
$\mathrm{NA}$ basal $(\mu \mathrm{g} / \mathrm{kg} / \mathrm{min})$ & $0,54 \pm 0,29$ & $0,56 \pm 0,39$ & 0,8 \\
$\mathrm{NA}$ a las $24 \mathrm{~h}(\mu \mathrm{g} / \mathrm{kg} / \mathrm{min})$ & $0,38 \pm 0,36$ & $0,35 \pm 0,44$ & 0,28 \\
NA diferencia $(\mu \mathrm{g} / \mathrm{kg} / \mathrm{min})$ & $0,16 \pm 0,32$ & $0,21 \pm 0,41$ & 0,44 \\
Diferencia de cortisol & 17 & 23 & \\
$\quad>9 \mu \mathrm{g} / \mathrm{dl}$ tras HDCST, $\mathrm{H}$ & & & \\
NA basal $(\mu \mathrm{g} / \mathrm{kg} / \mathrm{min})$ & $0,70 \pm 0,46$ & $0,62 \pm 0,31$ & 0,85 \\
NA a las $24 \mathrm{~h}(\mu \mathrm{g} / \mathrm{kg} / \mathrm{min})$ & $0,45 \pm 0,43$ & $0,43 \pm 0,40$ & 0,96 \\
NA diferencia $(\mu \mathrm{g} / \mathrm{kg} / \mathrm{min})$ & $0,25 \pm 0,43$ & $0,18 \pm 0,31$ & 0,45 \\
\hline
\end{tabular}

Grupo A: pacientes tratados con corticoides; grupo B: grupo control.

arterial aumentando la respuesta a la angiotensina II, la adrenalina y la noradrenalina ${ }^{2-6,12,20}$. Las guías de la Surviving Sepsis Campaign apoyan el uso de la LDST en el shock séptico dependiente de fármacos vasoactivos $^{1}$, independientemente del diagnóstico bioquímico $^{21}$, basándose en estudios aleatorizados donde el uso de hidrocortisona se ha asociado con menos tiempo de shock ${ }^{5-8,22}$ y disminución de la mortalidad $^{7}$. Sin embargo, su uso indiscriminado ha sido criticado $^{4,10,12}$, y en nuestra experiencia no se ha relacionado con una disminución del tiempo de shock -que sí se relacionó con la gravedad del cuadro- ni con una disminución de la mortalidad ${ }^{14}$. Por ello, determinar qué grupo de pacientes con shock séptico podrían beneficiarse del tratamiento con LDST es fundamental y sigue sin ser dilucidado ${ }^{10}$. No se debe olvidar tampoco los potenciales efectos adversos asociados al uso de corticoides en los pacientes en la $\mathrm{UCI}^{23}$.

Teóricamente, los pacientes con shock séptico que presentaran bajas cifras basales de cortisol o no respondieran a la estimulación suprarrenal deberían presentar una mejor respuesta a la LDST. Sin embargo, en nuestro estudio no hemos evidenciado un beneficio inmediato en la evolución hemodinámica (disminución de las dosis de NA a las 24 h) ni en la mortalidad; al igual que Rady et $\mathrm{al}^{24}$, Keh et $\mathrm{al}^{6}$ tampoco hallaron diferencias tras 24 h en la dosis de fár-

TABLA 5. Tratamientos aplicados a los pacientes en shock séptico

\begin{tabular}{|l|c|c|c|}
\hline & $\begin{array}{c}\text { Grupo A } \\
(\mathrm{n}=48)\end{array}$ & $\begin{array}{c}\text { Grupo B } \\
(\mathrm{n}=48)\end{array}$ & $\mathrm{p}$ \\
\hline $\begin{array}{c}\text { Ventilación mecánica, } \\
\mathrm{n}(\%)\end{array}$ & $44(91,7)$ & $43(89,6)$ & 1 \\
$\begin{array}{l}\text { Hemodiafiltración continua, } \\
\mathrm{n}(\%)\end{array}$ & $15(31,3)$ & $18(37,5)$ & 0,52 \\
$\begin{array}{l}\text { Drotrecogina alfa, n (\%) } \\
\text { Antibiótico inapropiado, } \\
\mathrm{n}(\%)\end{array}$ & $2(4,2)$ & $5(10,4)$ & 0,21 \\
$\begin{array}{c}\text { Balance hídrico a las 24 h (1), } \\
\text { media } \pm \text { DE }\end{array}$ & $2,7 \pm 2,3$ & $2,9 \pm 2,6$ & 0,59 \\
\hline
\end{tabular}

Grupo A: pacientes tratados con corticoides; grupo B: grupo control. 
macos vasoactivos en los pacientes con shock séptico tratados o no con corticoides. Los hallazgos de nuestro estudio no respaldan lo propugnado por algunos autores que indican que una respuesta hemodinámica precoz tras 1-2 días de tratamiento con LDST sería el punto crucial para continuar con dicho tratamiento ${ }^{10,12,13}$

Respecto a los parámetros bioquímicos que se asociarían teóricamente a una mejor respuesta hemodinámica al tratamiento, la controversia es aún mayor $^{10,12,21}$, dado que son bien conocidas las limitaciones de las pruebas diagnósticas en los enfermos críticos $^{25}$. Las cifras basales de cortisol consideradas «óptimas» en los pacientes críticos oscilan entre 10 y $34 \mu \mathrm{g} / \mathrm{dl}^{3,4,8,9,21}$. Ante la variabilidad de estas cifras, Marik et $\mathrm{al}^{26}$, en un estudio que incluyó a 59 pacientes con shock séptico, mostraron que cifras basales de cortisol de 23,7 $\mu \mathrm{g} / \mathrm{dl}$ serían el parámetro analítico más preciso para predecir la respuesta hemodinámica a la LDST. En nuestro estudio no hallamos diferencias en la respuesta hemodinámica de acuerdo con diferentes intervalos de cortisol basal. Sin embargo, debe reseñarse que el grupo de cortisol basal $<10 \mu \mathrm{g} / \mathrm{dl}$ sólo incluyó a 8 pacientes. Otros estudios recientes tampoco hallaron relación entre las cifras basales de cortisol, la respuesta hemodinámica y el pronóstico ${ }^{24,27}$.

En cuanto a la valoración de la reserva de la glándula suprarrenal, desde el estudio de Annane et $\mathrm{al}^{7}$, el test de estimulación suprarrenal con $250 \mu \mathrm{g}$ de corticotrofina se ha utilizado de modo generalizado. Sin embargo, esta prueba ha sido criticada, ya que supone un estímulo suprafisiológico de la glándula suprarrenal ${ }^{19}$. Por ello, algunos autores respaldan la práctica de estimulación suprarrenal con dosis bajas de corticotrofina ${ }^{28}$, que presenta una mayor sensibilidad diagnóstica, aunque no haya consenso respecto a su empleo en los pacientes críticos ${ }^{29}$. El estudio de Annane et $\mathrm{al}^{7}$ mostró una reducción de la mortalidad y el tiempo de shock sólo en los pacientes con ISR. Igualmente, Oppert et $\mathrm{al}^{8}$ mostraron que los pacientes con una inadecuada reserva suprarrenal presentaban una mejor respuesta hemodinámica cuando se los trataba con hidrocortisona, a diferencia del estudio de Bollaert et $\mathrm{al}^{22}$, que hallaron un beneficio hemodinámico en los pacientes sépticos con y sin ISR. Otros autores han señalado que la estimulación glandular no predice la respuesta hemodinámica y recomiendan el uso generalizado de corticoides ${ }^{30}$.

Nuestro estudio presenta varias limitaciones. La más importante es que se trata de un estudio retrospectivo con un número de pacientes pequeño. Sin embargo, los pacientes estaban bien apareados por diferentes cifras basales de cortisol y requerimientos de fármacos vasoactivos, y ambos grupos presentaban iguales características clínico-demográficas. Otra limitación es que no determinamos las cifras de globulina transportadora de esteroides. Aunque la concentración sérica de albúmina era similar en ambos grupos, no se pudo calcular el índice de cortisol libre, que constituye la porción biológicamente activa ${ }^{2,25}$.
Sin embargo, su uso en la clínica práctica actualmente es muy escaso ${ }^{19}$.

Concluimos que los pacientes con shock séptico tratados con LDST presentan a las $24 \mathrm{~h}$ una respuesta hemodinámica idéntica a la de quienes no reciben dicho tratamiento, independientemente de las cifras basales de cortisol y la respuesta a la estimulación suprarrenal.

\section{BIBLIOGRAFÍA}

1. Dellinger RP, Carlet JM, Masur H, Gerlach H, Calandra T, Cohen J, et al. Surviving Sepsis Campaign guidelines for management of severe sepsis and septic shock. Intensive Care Med. 2004:30:536-55

2. Marik PE. Mechanisms and clinical consequences of critical illness associated adrenal insufficiency. Curr Opin Crit Care. 2007;13:363-9.

3. Lamberts SW, Bruining HA, De Jong FH. Corticosteroid therapy in severe illness. N Engl J Med. 1997;337:1285-92.

4. Hamrahian A. Adrenal function in critically ill patients: How to test? When to treat? Cleve Clin J Med. 2005;72:427-32.

5. Briegel J, Forst H, Haller M, Schelling G, Kilger E, Kuprat G, et al. Stress doses of hydrocortisone reverse hyperdinamic septic shock: a prospective, randomized, double-blind, single-centre study. Crit Care Med. 1999;27:723-32.

6. Keh D, Boehnke T, Weber-Cartens S, Schulz G, Ahlers O, Bercker S, et al. Immunologic and hemodynamic effects of "lowdose" hydrocortisone in septic shock. A double-blind, randomized, placebo-controlled, crossover study. Am J Respir Crit Care Med. 2003;167:512-20.

7. Annane D, Sebille V, Charpentier C, Bollaert PE, Francois B, Korach JM, et al. Effect of treatment with low doses of hydrocortisone and fludrocortisone in patients with septic shock. JAMA. 2002;288:862-71.

8. Oppert M, Reinicke A, Gräf KJ, Barckow D, Eckardt KU. Plasma cortisol levels before and during "low-dose" hydrocortisone therapy and their relationship to hemodynamic improvement in patients with septic shock. Intensive Care Med. 2000;26: 1747-55.

9. Marik PE. Unraveling the mystery of adrenal failure in the critically ill. Crit Care Med. 2004;32:596-7.

10. Thys F, Laterre PF. Hydrocortisone in septic shock; too much, too little, too soon? Crit Care Med. 2005;33:2683-4.

11. Lipiner-Friedman D, Sprung CL, Laterre PF, Weiss Y, Goodman SV, Vogeser M, et al. Adrenal function in sepsis: the retrospective Corticus cohort study. Crit Care Med. 2007;35:1012-8.

12. Ligtenberg JJM, Zijlstra JG. The relative adrenal insufficiency syndrome revisited: which patients will benefit from lowdose steroids? Curr Opin Crit Care. 2004;10:456-60.

13. Beishuizen A, Thijs JG. Relative adrenal failure in intensive care: in identifiable problem requiring treatment? Best Pract Res Clin Endocrinol Metab. 2001;15:513-31.

14. Raurich JM, Llompart-Pou JA, Ibáñez J, Frontera G, Pérez O, García L, et al. Low dose steroid therapy does not affect hemodynamic response in septic shock patients: J Crit Care. 2007; 22:324-9.

15. Le Gall JR, Lemeshow S, Saulnier F. A new Simplified Acute Physiology Score (SAPS II) based on a European/North American multicenter study. JAMA. 1993;270:2957-63.

16. Knaus WA, Draper EA, Wagner DP, Zimmerman JE. APACHE II: a severity of disease classification system. Crit Care Med. 1985;13:818-29.

17. Vincent JL, Moreno R, Takala J, Willats S, De Mendonca A, Bruining H, et al; on behalf of the Working Group on SepsisRelated Problems of the European Society of Intensive Care Medicine. The SOFA (Sepsis-related Organ Failure Assessment) score to describe organ dysfunction/failure. Intensive Care Med. 1996;22:707-10.

18. $2001 \mathrm{SCCM} / \mathrm{ESICM} / \mathrm{ACCP} / \mathrm{ATS} / \mathrm{SIS}$ International Sepsis Definitions Conference. Crit Care Med. 2003;31:1250-6. 
19. De Jong MFC, Beishuizen A, Groeneveld ABJ. Defining relative adrenal insufficiency in the critically ill: The ACTH test revisited. En: Vincent JL, editor. Yearbook of intensive care and emergency medicine. Heidelberg: Springer; 2006. p. 539-50.

20. Prignent H, Maxime V, Annane D. Science review: Mechanisms of impaired adrenal function in sepsis and molecular actions of glucocorticoids. Crit Care. 2004;8:243-52.

21. Thomas Z, Fraser GL. An update on the diagnosis of adrenal insufficiency and the use of corticotherapy in critical illness. Ann Pharmacother. 2007;41:1456-65.

22. Bollaert PE, Charpentier C, Levy B, Debouverie M, Audibert G, Larcan A. Reversal of late septic shock with supraphysiological doses of hydrocortisone. Crit Care Med. 1998;26:645-50

23. Rady MY, Johnson DJ, Patel B, Larson J, Helmers R. Corticosteroids influence the mortality and morbidity of acute critical illness. Crit Care. 2006; 10:R101.

24. Rady MY, Johnson DJ, Patel B, Larson J, Helmers R. Cortisol levels and corticosteroid administration fail to predict mortality in critical illness: The confounding effects of organ dysfunction and sex. Arch Surg. 2005;140:661-8.
25. Arafah BM. Hypothalamic pituitary adrenal function during critical illness: limitations of current assessment methods. J Clin Endocrinol Metab. 2006;91:3725-45.

26. Marik PE, Zaloga GP. Adrenal insufficiency during septic shock. Crit Care Med. 2003;31:141-5.

27. De Jong MF, Beishuizen A, Spijkstra JJ, Groeneveld AB. Relative adrenal insufficiency as a predictor of disease severity, mortality, and beneficial effects of corticosteroid treatment in septic shock. Crit Care Med. 2007;35:1896-903.

28. Siraux V, De Backer D, Yavalatti G, Melot C, Gervy C, Mockel J, et al. Relative adrenal insufficiency in patients with septic shock: comparison of low-dose and conventional corticotrophin tests. Crit Care Med. 2005;33:2479-86.

29. Annane D. Low-dose adrenocorticotropic hormone test is not ready for routine adrenal function testing in the intensive care unit. Crit Care Med. 2005;33:2688-9.

30. Morel J, Venet C, Donati Y, Charier D, Liotier J, FrereMeunier D, et al. Adrenal axis function does not appear to be associated with hemodynamic improvement in septic shock patients systematically receiving glucocorticoid therapy. Intensive Care Med. 2006;32:1184-90. 